Meta

Journal des traducteurs

Translators' Journal

\title{
Collocations rythmologiques
}

\section{Gertrud Gréciano}

Volume 42, numéro 1, mars 1997

Lexicologie et terminologie

URI : https://id.erudit.org/iderudit/002965ar

DOI : https://doi.org/10.7202/002965ar

Aller au sommaire du numéro

\section{Éditeur(s)}

Les Presses de l'Université de Montréal

ISSN

0026-0452 (imprimé)

1492-1421 (numérique)

Découvrir la revue

Citer cet article

Gréciano, G. (1997). Collocations rythmologiques. Meta, 42(1), 33-44.

https://doi.org/10.7202/002965ar
Résumé de l'article

Le projet CONPHRAS-PROCOPE sert de cadre empirique, il vise la saisie bilingue (allemand-français) des locutions terminologiques, et se destine à la rédaction et à la traduction de textes spécialisés. Ici, deux domaines sont plus particulièrement explorés : la médecine et les institutions. Trois classes seront dégagées : les phraséotermes, les phrasèmes de spécialité et les phraséotextes. Des hypothèses sont formulées au sujet du partage des rôles. La complexité des phénomènes à dénommer réclame cette appellation polylexicale qui joue de façon préférentielle sur les registres général et spécial. Responsables de la phraséolo-gisation des termes, les collocataires se repèrent électroniquement, se représentent lexico-graphiquement et s'expliquent par leur dimension aussi bien nolionnelle que discursive. Vue sous son aspect collocalionnel, la langue spécialisée se révèle plus naturelle que formelle, ce qui n'est pas sans conséquences didactiques, voire dictionnairiques, puisqu'une complémentarité entre langues courante et savante s'impose.
Ce document est protégé par la loi sur le droit d'auteur. L'utilisation des services d'Érudit (y compris la reproduction) est assujettie à sa politique d'utilisation que vous pouvez consulter en ligne.

https://apropos.erudit.org/fr/usagers/politique-dutilisation/ 


\title{
COLLOCATIONS RYTHMOLOGIQUES*
}

\author{
GERTRUD GRÉCIANO \\ Université des sciences humaines de Strasbourg, Strasbourg, France
}

\begin{abstract}
Résumé
Le projet CONPHRAS-PROCOPE sert de cadre empirique, $i l$ vise la saisie bilingue (allemand-français) des locutions terminologiques, et se destine à la rédaction et à la traduction de textes spécialisés. Ici, deux domaines sont plus particulièrement explorés: la médecine et les institutions. Trois classes seront dégagées : les phraséotermes, les phrasèmes de spécialité et les phraséotextes. Des hypothèses sont formulées au sujet du partage des rôles. La complexité des phénomènes à dénommer réclame cette appellation polylexicale qui joue de façon préférentielle sur les registres général et spécial. Responsables de la phraséologisation des termes, les collocataires se repèrent électroniquement, se représentent lexicographiquement et s'expliquent par leur dimension aussi bien notionnelle que discursive. Vue sous son aspect collocationnel, la langue spécialisée se révèle plus naturelle que formelle, ce qui n'est pas sans conséquences didactiques, voire dictionnairiques, puisqu' une complémentarité entre langues courante et savante s'impose.
\end{abstract}

\begin{abstract}
Using the empirical framework of the CONPHRAS-PROCOPE project, our aim is to gather bilingual (German-French) terms and expressions for writing and translating specialized texts. Focussing primarily on the areas of medicine and the institutions, we have adopted multiple designations to identify the three types of phenomena observed - phrase-terms, specialized phrasemes and phrase-texts - and have formulated hypotheses as to their overlapping roles. Collocations are identified electronically, assigned to lexicons, and envisaged from both a conceptual and discoursal standpoint. From a collocational point view, specialized language more closely resembles natural language than formal language. The implications for didactics as well as for dictionary-writing are therefore significant as this beckons the need to recognize the complementarity of everyday language and specialized language.
\end{abstract}

Dans l'interdisciplinarité sous-jacente à ce colloque, entre les divers domaines, la linguistique et l'informatique, j'adopterai la perspective du linguiste et reviendrai sur des aspects importants de la polylexicalité terminologique, au cœur déjà des débats du colloque de Genève, publié dans Terminologie \& traduction (1992). Les preuves en faveur de la combinatoire syntagmatique ne sont définitivement plus à fournir; pour la langue générale, Mel'čuk (1994) l'estime à $40 \%$ dans le texte et à $90 \%$ dans le lexique; pour les vocabulaires scientifiques et techniques, Goffin (1992) la situe à $80 \%$. Si besoin était, ces relevés statistiques pourraient à eux seuls expliquer la présence de plus en plus forte de la phraséologie au sein des activités linguistiques.

Renouant avec le dialogue de Genève et ses interlocuteurs présents également à Lyon, on s'intéressera à la terminologie essentiellement polylexicale, en s'interrogeant sur sa nature savante ou courante et en examinant l'articulation entre langues spécialisée et générale, configurée en phraséologie scientifique. On vérifiera les derniers acquis de la phraséologie générale sur les plans cognitif, lexicographique et discursif en vue de leur adéquation explicative à la phraséologie spécialisée, en se servant de quatre mots clés: termes-vedettes (1), définitions (2), rôles (3) et images (4). Les deux premiers rejoignent la réflexion sur les dictionnaires, les deux derniers relèvent de la sémantique du lexique. 
La recherche phraséologique s'est concentrée sur la nature et la productivité de ses formatifs. Si, en phraséologie générale, la polylexicalité s'étend sur des constituants de langue commune: faire des pieds et des mains, la phraséologie spécialisée n'est pas pour autant le produit de formatifs exclusivement savants: les banquiers révisent/revoient à la baisse, tablent sur la croissance; la chute du titre et la faiblesse du dollar ( $\ll$ Revue des valeurs», Le Monde 20/21 août 1995). Dans le cadre du projet CONPHRASPROCOPE (Gréciano et Rothkegel 1996), la cohabitation entre langue générale et langue spécialisée se dégage comme régularité des cinq domaines étudiés: la médecine, l'économie, le sport, l'administration et la politique. Actuellement, une comparaison interdisciplinaire permet de reconnaître une graduation entre domaines à phraséologie plus ou moins technique :

(1)

+ degrés de technicité -

médecine, économie, sport, administration, politique.

Les status locutoires et illocutoires permettent de comprendre l'échelle scalaire, puisque, de proportion inverse, description référentielle et impact communicatif déterminent les extrêmes. La technicité résulte du figement phraséologique des formatifs, des rôles propres à un domaine et de leur hiérarchisation spécifique.

Les observations suivantes concernent la médecine. L'hypothèse de départ sur l'interaction plus ou moins grande et plus ou moins motivée de formatifs savants et courants au sein d'une terminologie sera examinée à travers la rythmologie cardiaque. Vérificateur et bénéficiaire, cette discipline, un sous-/microsystème médical, est en voie de devenir écosystème (Blampain 1992) avec la structuration du domaine selon l'anatomopathologie, l'électrophysiologie et la thérapeutique des troubles du rythme du cœur. Branche cardiologique de pointe, elle est née, s'est constituée et développée durant ces 25 dernières années avec les nouvelles techniques d'exploration, ce qui se répercute au niveau de sa terminologie. Elle offre un objet d'étude idéal et réclamé par le linguiste, une discipline jeune avec ses besoins spécifiques en dénomination et en textualisation.

\section{TERMES-VEDETTES}

La visualisation étant l'une des pratiques en médecine aussi bien qu'en linguistique, la seule saisie optique de l'un des premiers index rythmologiques, de Tricot et al. (1974) - retenu ici pour illustrer une terminologie naissante - confirme la rareté des termes techniques monolexicaux $(<5 \%)$. La complexité des phénomènes à dénommer réclame une appellation polylexicale donc phraséologique, ce qui est confirmé par l'augmentation des termes polylexicaux (environ $15 \%$ ) dans le plus récent des dictionnaires rythmologiques (Pelletier 1994).

Les termes monolexicaux sont des noms génériques qui restent des outils d'approximation, que seule la spécification par collocataires rend contextuellement opératoires. Ainsi, l'insensibilité à la collocation, voire une combinatoire syntagmatique zéro, est l'indice langagier de la non-productivité thématique des monotermes, comme systole, diastole, asystole, et explique leur quasi-absence dans l'index et dans le dictionnaire en question. Cette non-productivité peut s'expliquer scientifiquement: le trait distinctif de la rythmologie, outre la pathologie propre à toute discipline médicale, relève des techniques d'exploration; la rythmologie ne se réfère qu'indirectement à l'anatomie. Pour cette raison, extrasystole et tachycardie deviennent thématiquement porteurs, ce qui se traduit phraséologiquement par une combinatoire figée avec des collocataires. De ce fait, des termes monolexicaux se solidarisent en termes polylexicaux, en phraséotermes, puisque le collocataire devient responsable de la phraséologisation du terme: 
(2) anévrisme

arythmie extrasystole bradycardie tachycardie

\author{
auriculaire / ventriculaire \\ sinusale / auriculaire / jonctionnelle / ventriculaire \\ auriculaire / jonctionnelle / ventriculaire \\ sinusale / auriculaire / jonctionnelle / ventriculaire \\ sinusale / auriculaire / jonctionnelle / ventriculaire.
}

Est lexicalisée l'affinité élective de la dénomination descriptive du symptôme pathologique, assurée par la base/le noyau/le collocateur, avec l'ancrage anatomique, technologique, pharmacologique, assuré par le collocataire. Ce sont les collocataires qui garantissent la démarche proprement scientifique à travers la spécification. Il y a liage notionnel entre collocateurs et collocataires. Les deux procédures, la dénomination pathologique et les spécifications diverses, participent activement à la description référentielle, reconnue décisive pour le degré de technicité du discours médical, la référentialisation dominant dans le collocataire, la description dans le collocateur du phraséoterme.

$\mathrm{Si}$, en raison de leur carence en dénomination pathologique, les termes diastole et systole ne sont pas productifs en tant que collocateurs, ils peuvent intervenir constructivement en tant que collocataires et jouent pleinement alors leur rôle spécificateur, non pas anatomique, mais procédural. Le symptôme pathologique est localisé dans le collocateur, la spécification réfère aux deux phases de l'activité du cœur, sa contraction et son relâchement:

\title{
(3) bruit systolique/diastolique temps systolique / diastolique
}

Ces collocataires en antonymie converse: sinusal/atrial/jonctionnel/ventriculaire (2) et systolique/diastolique (3) confirment pour la phraséologie spécialisée les moules générateurs / Phraseoschablonen, bien connus dans la phraséologie générale, des structures figées sous-jacentes à certains phrasèmes, qui, tout en laissant une apparente liberté lexicale, réclament un nombre fixe de cooccurrents et leur interrelation déterminée, ici l'antonymie.

Certes, la productivité des formatifs savants existe (2), elle est accentuée dans Pellerin (1994). Dans Tricot et al. (1974), elle reste mesurée encore, comparée à celle des formatifs courants qui l'emportent. L'appellation polylexicale joue de façon préférentielle sur le registre général et le registre spécial. Les exemples (3) annoncent la longue liste des collocateurs et le nombre limité des collocataires, tous deux emprunts à la langue générale.

Sur les 110 termes de rythmologie répertoriés, l'index de Tricot et al. (1974) atteste des formatifs courants pour 77 collocateurs et 24 collocataires, des formatifs savants pour 33 collocateurs et 86 collocataires. Sur les 77 collocateurs courants, 57 sont colloqués de formatifs savants et 20 de formatifs courants. Sur les 33 collocateurs savants, 29 sont colloqués de formatifs savants et 4 de formatifs courants. Selon ces données, on peut conclure à une participation de la langue générale à 101 et de la langue spécialisée à 109 termes rythmologiques. La combinatoire entre collocateur courant et collocataire savant s'avère la plus productive:

(4)

collocateurs
collocataires

(5)

collocateurs courants collocateurs savants

formatifs courants
$70 \%$
$23 \%$
collocataires savants
$52 \%$
$26 \%$
formatifs savants
$30 \%$
$87 \%$
collocataires courants
$18 \%$
$4 \%$

Si la médecine contemporaine est habituée aux formatifs spécialisés, le linguiste et à ce propos, terminologue et phraséologue se rejoignent - note la forte présence des 
formatifs généraux dans le corpus étudié. Voici un échantillon des quatre combinatoires autour de formatifs généraux et spéciaux, extrait de Tricot et al. (1974), en privilégiant chaque fois la première.

(6) Collocateurs courants avec:

- collocataires savants :

activité auriculaire, arrêt sinusal/cardiaque, bloc auriculoventriculaire, déficience sinusale, dissociation auriculoventriculaire, faisceau de His/James/Kent/Mahaim, foyer ectopique, fusion auriculaire / ventriculaire, pause sinusale / auriculaire / ventriculaire, rythme auriculaire / jonctionnel/ventriculaire, trouble de la repolarisation ventriculaire:

collocataires courants: aberration de conduction, bloc à deux étages, bloc de branche droit/gauche, bloc d'entrée / de sortie, conduction bloquée/cachée, pause auriculaire / ventriculaire, repos compensateur, trouble de conduction, trouble du rythme, rythme réciproque, torsade de pointe.

(7) Collocateurs savants avec:

- collocataires savants:

anévrisme auriculaire / ventriculaire, arythmie sinusale / auriculaire / jonctionnelle / ventriculaire;

collocataires courants: arythmie (in)complète, tachycardie double/irrégulière/ lente.

\section{DÉFINITIONS}

Si la macrostructure d'un répertoire/glossaire s'avère informative quant à la productivité et à la nature des vedettes et quant à la structuration du domaine correspondant, ce qui vient d'être exploré dans les index, la microstructure d'un dictionnaire n'est pas moins riche en renseignements. À ce propos, Garnier et Delamare (1989) offrent un corpus intéressant. La langue naturelle y est à l'honneur, ce qui donne à l'ouvrage sa marque culturelle et discursive; voué d'une part à la francophonie dans la tradition académique, il se destine d'autre part à la médiation lexicographique en respectant les locutions consacrées par l'usage. Les définitions, particulièrement révélatrices quant à l'impact de la langue générale sur la langue spécialisée, sont une ressource complémentaire pour saisir les procédures cognitives engagées. La définition est au service de la découverte et de l'anticipation de régularités, de la constitution notionnelle, de la progression scientifique donc. La définition se destine au transfert du savoir; explicative et persuasive, elle pose, décrit et impose les réalités en question. Le sémanticien la souhaite naturelle (Martin 1990), le logicien assertive (Tarski 1936), le pragmaticien normalisatrice (Wittgenstein 1969), le terminologue référentielle (de Bessé 1990) et performative (Rey 1979). Comme elle vise la formulation langagière de l'ensemble des phénomènes nécessaires à un domaine (Pawlowski 1980), elle est constitutive du discours lexicographique.

Selon la nature des choses, les définitions des termes médicaux sont dictionnairiques et encyclopédiques. On constate une grande convergence entre les vedettes des registres respectifs. La divergence réside dans les modes d'explication, assumée par la visualisation électrocardiographique commentée chez Tricot et al. (1974), par la définition chez Garnier et Delamare (1989); support technique d'une part, support langagier d'autre part. Si l'on vérifie les termes de l'index quant à leurs définitions dictionnairiques, de grands champs synonymiques et paraphrastiques du domaine s'ouvrent là où - fonction discursive, voire communicative oblige - la langue générale l'emporte :

(8) arrêt cardiaque : cessation des contractions du cour, arrêt de la circulation, disparition de la pression artérielle; 
(9) bloc... suivi de 49 collocataires dont 43 termes cardiologiques: état pathologique empêchant la diffusion..., maladie dégénérative limitée à..., associant une atteinte.. entraînant une attitude vicieuse..., trouble du rythme cardiaque caractérisé par... trouble de la conduction comportant l'atteinte..., variété de bloc... située..., dans laquelle la lésion siège.

(10) arythmie : anomalie du rythme... désigne surtout les perturbations du rythme, irrégularité du rythme... accompagnée de..., provoquée par..., peut intervenir dans...

(11) conduction (trouble de) : anomalie du rythme cardiaque due à... née de... se traduit par... qui provoque souvent...

(12) systole : contraction du muscle cardiaque

diastole : relâchement du cour... correspond au grand silence

asystole : arrêt des contractions cardiaques... immobilité

extrasystole : contraction supplémentaire

bradycardie : ralentissement des battements du cour.

tachycardie : accélération du rythme des battements cardiaques

(13) torsade de pointe : trouble... consistant en... caractérisé... spontanément curable... il provoque... survient.

Il est intéressant d'observer qu'à son tour, la collocation définitoire explicite et amplifie les fonctions inférées par le phraséoterme, en sollicitant l'activité langagière à travers les compétences lexicales et syntaxiques de la langue générale: synonymie, détermination, qualification, relation spatiale et causale. La définition peut être vue comme une didactisation de la procédure terminologique, médiation entre le terme et le texte; elle assume :

- la signalisation de la pathologie par des collocateurs nominaux à $2 / 3$ courants : état pathologique/maladie dégénérative/anomalie/irrégularité/cessation/ disparition/ralentissement/ accélération/ dérangement/ trouble / perturbation/ arrêt/ contraction supplémentaire...

- sa description explicative par des formatifs prédicatifs nominaux, adjectivaux et verbaux colloqués, qui sont courants dans la définition et savants dans le terme: arrêt de la circulation / disparition de la pression artérielle / comportent une atteinte / entraînent une attitude / empêchant la diffusion / accompagné de / provoqué par/caractérisé par / dû à / né de / se traduit par / provoque souvent/survient / consiste en.

Ce qui est intéressant en cette phase de la définition et inexistant et inconcevable au niveau du terme aussi bien qu'au niveau de la signalisation de la pathologie, ce sont les traces d'évaluation qui traduisent le jugement, l'expérience et l'autorité de l'auteur et rendent la description explicative. Soudain surgit le locuteur-médecin, qui répond aux attentes d'anticipation et de pronostic du lecteur/usager: peut intervenir dans/regrettable! spontanément curable/ sans gravité;

- sa localisation par des prédicats adjectifs colloqués et verbaux collocateurs: systolique, diastolique, droit, gauche, à deux étages, être limité à / associant une atteinte à / être situé à / dans laquelle la lésion siège.

La définition elle-même se révèle un moule discursif, un phraséotexte: polylexicale et figée, avec son lexique et sa structure propre. Les vedettes et la signalisation définitoire représentent des locutions exclusivement nominales; aux deux dernières phases définitoires, la description et la localisation, apparaît le verbal, des formules consacrées génératrices de texte. La définition est tenue au respect des conditions requises afin d'être 
scientifiquement opératoire. Il y a beau y avoir équivalence référentielle entre la vedette et la définition, seule la vedette répond aux exigences théoriques et pratiques propres à la terminologie: concentration/condensation/cristallisation notionnelle d'une part, pertinence et efficacité communicative d'autre part. Le respect des dimensions logiques et pragmatiques réserve à lui seul le pouvoir de nomination. De la comparaison de la vedette avec sa définition ressort clairement la valeur terminologique des formatifs, courants aussi des vedettes. Le liage distributionnel et contextuel, la combinatoire leur confère une valeur savante.

\section{RÔLES}

Depuis peu, la phraséologie générale travaille volontiers sur les rôles figés dans les locutions; ils sont de nature argumentale et se trouvent en valence sémantique; on les dégage des textes littéraires et utilitaires pour reconstituer la mise en scène thématique du système phraséologique. CONPHRAS-PROCOPE applique cette démarche à la langue spécialisée. Delplanque-Tchamitchian (1995), Drillon (1995) et Gautier (1995) mettent en relief les scénarios propres à l'économie, au sport et à la politique. Pour le moment, cette dernière se révèle être le domaine le moins typique et le plus proche de la langue générale.

La dimension phraséologique des termes rythmologiques et de leurs définitions permet de dégager les rôles sémantiques qui sont les acteurs thématiques de ce domaine. Ils réfèrent phénoménologiquement en renvoyant à l'anatomopathologie, à l'électrophysiologie et à la thérapie des troubles du rythme. L'analyse des rôles intéresse les collocations terminologiques aussi bien que définitoires. Après les premières analyses qui sont encore à compléter, à vérifier et à systématiser, les formatifs représentent la diversité des actions, états, procès et propriétés incriminés.

Les formatifs courants - ce sont eux qui dominent dans l'index et dans les définitions dictionnairiques - représentent pour la plupart les prédicats nominaux des termes vedettes et les prédicats verbaux des définitions. Ils modélisent les attributs en exprimant des:

(14) actions: activités;

procès : accélération, cessation, conduction, contradiction, disparition, fusion, relâchement, rythme; empêcher, entraîner, limiter;

états : arrêt, état, bloc, pause, immobi lité ;

propriétés : anomalies, déficiences, faisceau, foyer, perturbation, trouble, aberration, torsade; caractériser, comporter.

La consignation n'est pas toujours sans problème, et la fréquence est significative. L'action, par définition intentionnelle et télique, ne convient qu'exceptionnellement à la nature organique. La spécificité du domaine semble se manifester dans la prédominance des procès et états et de leur combinatoire avec des propriétés. En effet, la médecine thématise la modalité irrégulière, anormale, bref pathologique des phénomènes.

Les collocataires rythmologiques épithètes, composés et élargissements nominaux, se satisfont plus ou moins bien des rôles répertoriés depuis Fillmore (1968) jusqu'à von Polenz (1985) pour la langue générale. Leurs attributions dépassent le savoir linguistique et réclament les connaissances médicales, car le cour et ses parties ne sont pas seulement LOCATIF, donc le lieu des événements, mais deviennent le carrefour, le théâtre du «petit drame» dans son ensemble (Tesnière): PATIENT ou OBJET AFFECTÉ d'une pathologie, INSTRUMENT d'une technique, BÉNÉFICIAIRE d'une thérapie. Par le biais de paraphrases et de transformations - résultant d'une étroite coopération technolinguistique car leur acceptabilité sémantique doit être contrôlée par les spécialistes, linguistes et médecins - , on obtient une première attribution des fonctions. Il importe de rappeler que 
ce partage des rôles des collocataires majoritairement savants est tributaire de la base collocatrice courante.

(15) AGENTS : activité auriculaire, bloc de branche

Paraphrase : l'oreillette est active ; la branche ne conduit plus...

PATIENTS : arrêt sinusal/cardiaque, déficience sinusale

Paraphase : le sinus / le cour est arrêté/est déficient...

EXPÉRIENTS : dissociation auriculo-ventriculaire, trouble de conduction, trouble du rythme

Paraphrase: la jonction auriculo-ventriculaire est frappée par une dissociation; la conduction/le rythme est frappé(e) par des troubles...

INSTRUMENTS : rythme auriculaire/jonctionnel/ventriculaire, pause sinusale/ auriculaire / ventriculaire

Paraphrase: l'activité est régulière et périodique dans l'oreillette/... l'oreillette permet d'enregistrer une activité régulière et périodique...

LOCATIFS : bloc de branche gauche/droit, bloc à deux étages

Paraphrase : à gauche / droite la branche ne conduit plus...

OBJETS AFFECTÉS : trouble de la repolarisation ventriculaire, dissociation auriculoventriculaire

Paraphrase : le trouble affecte la repolarisation du ventricule... la jonction auriculoventriculaire est affectée / frappée par une dissociation...

TEMPS : bloc d'entrée / de sortie

Paraphrase : au moment d'entrer/de sortir...

CAUSE / EFFET : provoque, être provoqué... intoxication à la digitaline

Paraphrase : cet accès provoque une crise d'angine de poitrine...

Des concordances déjà se manifestent: la rareté des actions s'accompagne d'un nombre peu élevé d'AGENTS; à l'inverse, les PATIENTS, OBJETS AFFECTÉS et EXPÉRIENTS sont nombreux, en écho aux procès et états qui prédominent en rythmologie. Certes, la localisation est importante, car les aspects anatomiques restent typiquement associés aux troubles du rythme cardiaque. Cependant, des compléments s'imposent. Une recherche encyclopédique aussi bien que linguistique est nécessaire, si l'on doit distinguer de façon plus nette entre LOCATIF et notamment INSTRUMENT, entre PATIENT, EXPÉRIENT et OBJET AFFECTÉ, entre PATIENT et AGENT. Probablement là aussi, la spécificité du domaine exige l'adaptation des catégories conçues pour la langue générale aux modes de représentation des connaissances rythmologiques.

\section{IMAGES}

Si les formatifs savants contribuent à la spécification notionnelle des collocations terminologiques, de nombreux formatifs courants procèdent à la modélisation analogique. Plusieurs travaux décrivent les analogies et métaphores scientifiques (Perelman 1969; Vigh 1975; Demandt 1978; Molino 1979; Gréciano 1983; Rigotti 1994; Hundt 1995). L'imagerie rythmologique se verbalise dans sa phraséologie, dans les collocations terminologiques et définitoires autour de formatifs généraux. Ces images langagières ont des convergences anatomopathologiques et électrophysiologiques et évoluent sur le canal optique, acoustique et tactile.

Dans cette spécialité, on est frappé par le grand nombre de métaphores optiques. Inspirées par la technique d'exploration, elles se lisent avant tout dans le tracé électrocardiographique :

(16) torsade de pointe, axe électrique du cour, segment ST en dôme / en cupule, flutter en dents de scie, fibrillation auriculaire à petits/gros grains/à larges mailles, rythme accordéon. 
À la différence de la médecine générale, peu d'images renvoient à l'anatomie :

(17) branche, faisceau, canal, atrium/oreillette, ventricule.

Les métaphores acoustiques sont perçues par la clinique, à l'auscultation, à la palpation ou par les appareils de physique:

(18) bruit irrégulier, salve d'extrasystoles, rafale d'extrasystoles.

La métaphore langagière, figée dans le formatif général, est plus souvent collocataire: pointe, axe, dôme, cupule, dents de scie, petits / gros grains, larges mailles que collocateur: bruit, salve, rafale.

La sémiologie médicale interprète ces formes, sons et mouvements comme indices et icônes motivés ou comme symboles arbitraires. Les phraséotermes sont une verbalisation de l'icône, du symbole ou des deux à la fois: bloc de branche. Il y a ressemblance entre la perception visuelle et phonique et le sens littéral des formatifs : bloc, branche. Le formatif illustre soit le symbole: bloc, optique dans l'ECG ou acoustique dans l'échocardiogramme, soit l'icône anatomique: branche. Mais le signe iconique aussi bien que symbolique sont tous les deux index de la réalité pathologique, les troubles du rythme. Les manuels s'inspirent de l'imagerie surtout électrographique, donc codée et symbolique; parfois on la retrace dans sa variété. Pour bloc de branche, Gay et al. (1990) représentent le symbole dans l'ECG et l'icône dans le schéma anatomique. Puech et al. (1985) réunissent à leur façon l'ECG avec l'anatomie. Loin de l'ornement, l'analogie à l'œuvre dans le corpus rythmologique est descriptive. Elle doit sa force interprétative à la nature courante des formatifs concernés.

La dénomination analogique et sa terminologisation créent une similitude de fait entre le rythme cardiaque et l'univers de la géométrie, de la physique, de la botanique, de la technique, de l'architecture et de la géographie, qui peut être liée à la:

- forme : atrium, oreillette, ventricule, branche, torsade, faisceau;

- fonction : axe, bloc, entrée, sortie, extrasystole gachette, sentinelle, réciproque.

La pensée analogique pose des ressemblances, ce qui présuppose sélection et fiction, empirie et intuition; elle vise non pas l'esthétique mais l'utile. L'image est heuristique et mnémotechnique. Née d'un besoin de clarté, cette appellation est pertinente et efficace, car perceptible; elle confère à la langue de spécialité sa qualité (Oksaar 1986; Gauger 1986). Elle explique la popularité des atlas électrocardiographiques et le succès du répertoire bilingue illustré de l'anatomie humaine (Feneis et Dhem 1985).

La phraséologie contemporaine s'intéresse tout particulièrement aux domaines fournisseurs d'images. La nature organique et la technologie nourrissent les métaphores historiques et politiques (Gréciano 1994), les images zoologiques ont orienté la psychologie du comportement (Gréciano 1983 et 1992), la physique et la météorologie conditionnent la langue de l'économie et de la bourse (Cohen 1992; Hundt 1995). Si, dans certains contextes médicaux, les linguistes déplorent les emprunts militaires en raison de leurs modélisations cognitives inadaptées (Liebert 1994), on doit relever les ressources très évidentes de l'iconologie rythmologique.

Cette discipline conceptualise selon des procédures iconiques (rapport entre les signes linguistique et électro-échographique) et symboliques (rapport entre les signes électro-échographique et anatomo-pathologique). La représentation terminologique des connaissances opère avec des formatifs communs, relevant de l'expérience préconceptuelle et se contentant de ressemblance de famille (Lakoff 1987). Les sémantiques cognitives et mentales conviennent bien pour expliquer la constitution du savoir 


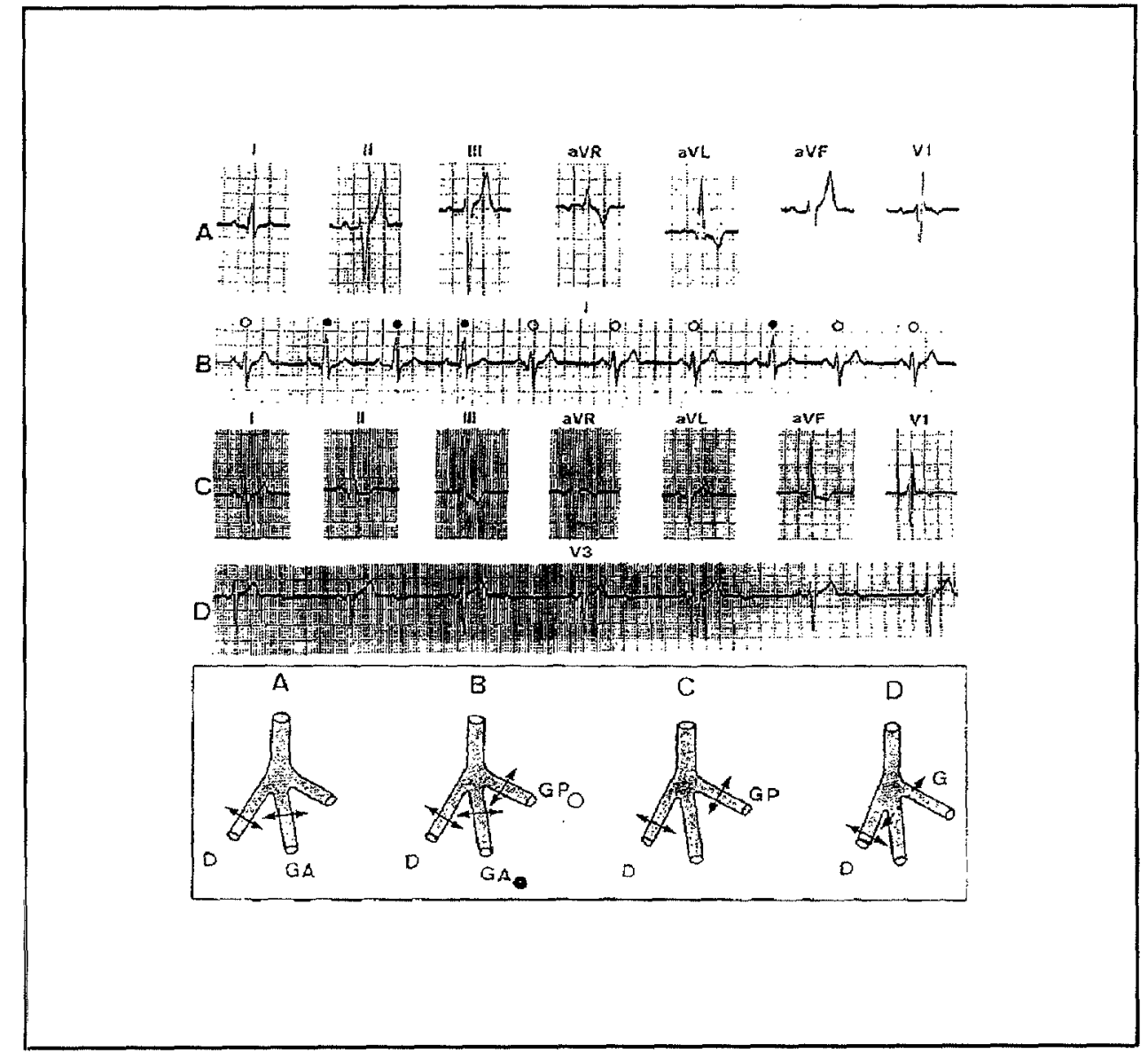

Figure 1:

Bloc trifasciculaire. Évolution chez un même patient

Tracé A :

Rythme sinusal: $\mathrm{PR}=0,21 \mathrm{~s}$ :

BBD (en VI : aspect rSR': DI : 0,12 s: QRS : $0,14 \mathrm{~s}$ ) et HBGA (en standard : AQRS : - 70 : qR en I, rS en II et III).

Tracé C:

PR : $0,16 \mathrm{~s}: \mathrm{BBD}$ (en VI : aspect rSR' : DI : $0,10 \mathrm{~s}:$ QRS : $0,14 \mathrm{~s}$ ) et HBGP (en standard : AQRS : + $100^{\circ}: \mathrm{qrS}$ en I (aspect atypique) et qR en II et III).

Tracé B :

Alternance sur le même enregistrement en I d'un aspect d'HBGP (avec PR à 0,16 s) et d'HBGA (avec PR à $0,20 \mathrm{~s}$ ). La variation de la durée de PR peut s'expliquer par une conduction relativement plus lente sur le faisceau postérieur de la branche gauche, traduite par un espace PR plus long en présence de l'HBGA.

Tracé D :

BBD (affirmé sur l'aspect rSr' en V3 et l'allongement de la durée de QRS) associé à un BAV du $2^{\mathrm{e}}$ degré type Möbitz: onde $\mathrm{P}$ bloquée une fois sur deux. Ce BAV de $2^{\mathrm{e}}$ degré siège sur la branche gauche du faisceau de His et non dans le tronc commun du faisceau de His ou le nœud d'Aschoff-Tawara, en raison des aspects précédemment enregistrés d'hémiblocs gauches antérieur et postérieur. 
rythmologique. Leur démarche prototypique et associative parvient à expliquer le réemploi préférentiel de représentants généraux en raison de leur typicalité : en dent de scie, à petits / gros grains, à larges mailles, constitutifs aussi de la géométrie, de l'architecture ou de 1'électricité: axe, bloc, cupule, dôme, segment, tracé, torsade, triangle, foyer, de l'acoustique : bruit, segment, salve, rythme, pause, rafale. L'expérience commune et technique est le fournisseur d'images rythmologiques, ce qui explique l'adéquation des modèles fondés davantage sur les primitifs, percepts et réseaux sémantiques que sur les traits distinctifs.

La dimension phraséologique du discours rythmologique autorise une série de conclusions. La collocation intervient activement au niveau de la dénomination terminologique et de sa définition; elle naît de la contiguïté entre langue de spécialité et générale et - effet de surprise pour une discipline hautement technologique - elle semble se complaire au contact des formatifs courants. La fonction des formatifs collocateurs et collocataires s'avère cognitivement fondatrice: rôles phénoménologiques, images heuristiques, de sorte que l'on doit à la polylexicalité terminologique la plus grande monosémie scientifique. Si les modèles cognitifs conçus pour la langue générale nécessitent des adaptations en vue de l'attribution des rôles en rythmologie, ils conviennent particulièrement bien à l'interprétation de ses images.

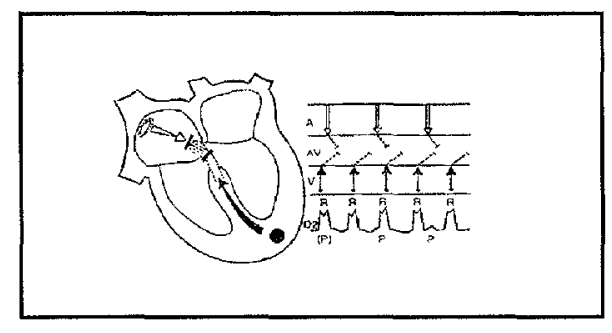

Figure 2 :

Schéma d'une tachycardie ventriculaire avec dissociation auriculoventriculaire complète, les oreillettes restant sous contrôle sinusal

Ainsi, cette analyse de la collocation rythmologique confirme plusieurs des hypothèses émises au colloque de Genève: la collocation comme mise en discours du terme (Béjoint et Thoiron 1992: 513); l'inséparabilité entre la terminologie et la phraséologie, le terme étant conditionné par le contexte (Reichling 1992: 486); le lexique général comme ressource de la terminologie (Goffin 1992:432); l'intelligibilité de la terminologie facilitée par sa nature générale (Goetschalckx 1992: 482).

Affaire de cour. Après avoir exploré les conceptualisations diverses du formatif cœur en phraséologie générale au colloque de Genève, je traite plus particulièrement dans la présente étude de la phraséologie cardiologique. Le bilan ne saurait être qu'une étape intermédiaire. Il y a d'abord la prise en compte de l'évolution de la terminologie médicale: deux réformes en quatre ans pour conformer le lexique français à la nomenclature internationale, elle-même en mutation. Le sommaire de Pellerin (1994) confirme la structuration du domaine sous-jacente à Tricot et al. (1974). Son index, par contre, réserve une 
place plus importante à la monolexicalité et complète les collocateurs actifs : canal, triangle, période, potentiel, préexcitation, électrocardiogramme, stimulateur, formatifs savants, marqués surtout par les techniques. Il ne renonce pas encore aux éponymes disparus dans la nomenclature internationalement uniformisée au bénéfice de spécifications anatomiques : faisceau de His et non pas faisceau atrio-ventriculaire.

CONPHRAS-PROCOPE continue à travailler à la gestion discursive et lexicographique correspondante, au repérage électronique des collocateurs et collocataires dans le texte, à leurs répertoires dictionnairiques mono- et plurilingues. Mais dès à présent, une révision des priorités didactiques s'impose, car la sémantique lexicale ne va plus sans sémantique combinatoire, et la sémantique spécialisée ne se passe pas de la sémantique générale. Pour la rythmologie ici examinée se dégage un travail cognitif partagé : les formatifs courants apportent une contribution essentielle à la sémantique des images aussi bien que des rôles, les formatifs savants assument, pour moitié, la précision de ces derniers. En effet, la compétence phraséologique peut guider la constitution du savoir encyclopédique et la langue spécialisée, qui, sous son aspect collocationnel, est plus naturelle que formelle, réclame une complémentarité entre les langues courante et savante. La coopération interdisciplinaire est définitivement nécessaire aux spécialistes aussi bien des domaines que de leur langue et de leur informatisation. La langue spécialisée est objet et objectif d'étude commun.

Notes

* Cet article est issu d'une communication présentée par l'auteur aux IVes Journées scientifiques du réseau «Lexicologie, terminologie, traduction» de l'AUPELF-UREF (Lyon, France, 28, 29, 30 septembre 1995).

\section{RÉFÉRENCES}

BÉJOINT, H. et Ph. THOIRON (1992) : «Macrostructure et microstructure dans un dictionnaire de collocations en langue de spécialité», Terminologie et traduction, 2/3, pp. 513-522.

DE BESSÉ, B. (1990) : «La définition terminologique», Chaurand et Mazière (dir.), La définition, Paris, pp. 252-261.

BLAMPAIN, D. (1992) : «Traduction et écosystème terminologique», Terminologie et traduction, 2/3, 457-466.

COHEN, B. (1992): «Méthodes de repérage et de classement des cooccurrents lexicaux», Terminologie et traduction, 2/3, pp. 505-511.

DELAMARE et al. (1975): Dictionnaire des maladies cardiovasculaires, Paris.

DELPLANQUE-TCHAMITCHIAN, C. (1955): Phraséologie et terminologie dans le discours économique allemand, Thèse de doctorat, Université de Strasbourg II.

DEMANDT, A. (1978): Metaphern für Geschichte. Sprachbilder und Gleichnisse im historisch-politischen Denken, München.:

DRILLON, M. L. (1995): Du style des figures aux figures de style: terminologie et phraséologie du patinage, Mémoire de DEA, Université de Strasbourg II.

FENEIS, H. et A. DHEM (1985) : Répertoire illustré d'anatomie humaine, Bruxelles.

FILLMORE, Ch. (1968): "The Case for Case», Bach et Harms (Eds), Universals in Linguistic Theory, New York, pp. 1-88.

GARNIER, M. et J. DELAMARE (1989) : Dictionnaire des termes de médecine, Paris.

GAUGER, H. M. (1986) : «Zur Sprache der Wissenschaft: sermo incurvatus in se ipsum», Kalvenkämper und Weinrich (Eds), Deutsch als Wissenschaftssprache, Tübingen, pp. 119-123.

GAUTIER, L. (1995): Les locutions à verbe support dans le discours public autrichien, Mémoire de DEA, Université de Strasbourg.

GAY, J. et al. (1990) : L'électrocardiogramme, Paris.

GHAZI, J. (1985) : Vocabulaire du discours médical, structure, fonctionnement, apprentissage, Paris.

GOETSCHALCKX, J. (1992) : «Terminologie et phraséologie», Terminologie et traduction, 2/3, pp. 477-484.

GOFFIN, R. (1992): «Du synthème au phraséolexème en terminologie différentielle», Terminologie et traduction, 2/3, pp. 457-466.

GRÉCIANO, G. (1983) : «Signification et dénotation en allemand. La sémantique des expressions idiomatiques», Recherches linguistiques $I X$, Paris.

GRÉCIANO, G. (1992) : «Zum System der Phrasemverwendung», Földes (Ed.), Deutsche Phraseologie in Sprachsystem und Sprachverwendung, Wien, pp. 149-169. 
GRÉCIANO, G. (1994) : «Fachphraseologie», Métrich und Vuillaume (Eds), Rand und band. Festschrift für Eugène Faucher, Tübingen, pp. 183-195.

GRÉCIANO, G. und A. ROTHKEGEL (1996) : «CONPHRAS-PROCOPE», Baur und Chlosta (Eds), Von der Wortmetapher zur Satzmetapher. Studien zur Phraseologie und Parömiologie 6, Bochum (in Drunck).

HUNDT, M. (1995): Modellbildung in der Wirtschaftssprache: zur Geschichte der Institutionen- und Theoriefachsprache der Wirtschaft, Tübingen.

LAKOFF, G. (1987): Women, Fire and Dangerous Things, Chicago,

LIEBERT, W. A. (1994) : «Lascaux - a Hypermedia Lexicon of Metaphor Models for Scientific Imagination», Martin et al. (Eds), Euralex 1994, Amsterdam, pp. 494-500.

MARTIN, R. (1990) : «La définition 'naturelle'», Chaurand et Mazière (dir.), La définition, Paris, pp. 86-96.

MELCUK, I. A. (1994): «Typologie des phrasèmes et leur présentation dans un dictionnaire de langue», Conférence plénière au colloque international La locution, Paris, ENS Saint-Cloud.

MOLINO, J. (1979) : «Métaphores, modèles et analogies dans les sciences», Langages, 54, pp. 83-102.

OKSAAR, E. (1986): «Gutes Wissenschaftsdeutsch - Perspektiven der Bewertung und der problemlösung», Kalverkämper und Weinrich (Eds), Deutsch als Wissenschaftssprache, Tübingen, pp. 100-118.

PAWLOWSKI, T. (1980): Begriffsbildung und Definition, Berlin.

PELLETIER, D. (1994) : Dictionnaire de rythmologie. Mots \& concepts, Paris.

PERELMANN, Ch. (1969): «Analogie et métaphore en sciences, poésie et philosophie», Revue internationale de philosophie, 87 (1), pp. 3-15.

PHAL, A. (1971) : Vocabulaire général d'orientation scientifique, Paris.

POLENZ, P. von (1985): Deutsche Satzsemantik. Grundbegriffe des Zwischen-den-Zeilen-Lesens, Berlin.

PUECH, P. et al. (1985) : Dysrythmies cardiaques, Paris.

REICHLING, A. (1992) : «Le traitement de la phraséologie dans EURODICAUTOM», Terminologie et traduction, $2 / 3$, pp. $485-492$.

REY, A. (1979): La terminologie: noms et notions, coll. «Que sais-je ?» $\mathrm{n}^{\circ} 1780$, Paris, PUF.

RIGOTTI, F. (1994) : Die Macht und ihre Metaphern. Über die sprachlichen Bilder der Politik, Paris.

SOURNIA, J. Ch. (1994) : «Les phases évolutives du vocabulaire médical français», Meta, 39 (4), pp. 692-700.

TARSKI, A. (1936) : «Der Wahrheitsbegriff in den formalisierten Sprachen, Studia philosophica, I.

THOIRON, Ph. (1994): «Résuitats de l'enquête d'implantation des termes de santê et de médecine», Terminologies nouvelles, 12, pp. 77-103.

TRICOT, R. et al. (1974): Atlas des troubles du rythme, Paris.

VIGH, A. (1975): «Comparaison et similitude», Le français moderne, 3, pp. 214-234.

WITTGENSTEIN, L. von (1969) : Philosophische Untersuchungen, Frankfurt. 